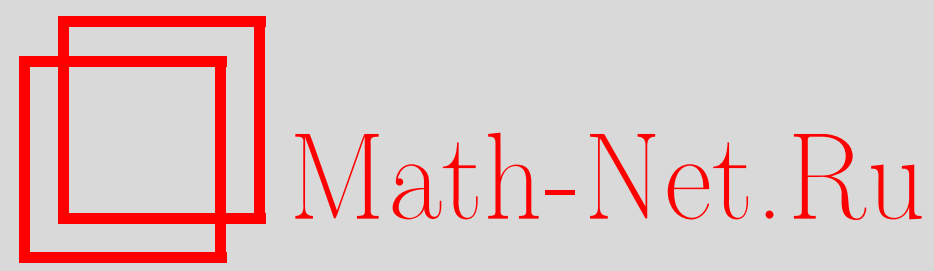

В. Г. Гольдштейн, Н. В. Сайдова, А. К. Танаев, Математическое моделирование продольных токов смещения и поверхностного эффекта в многослойной земле и проводах линий электропередачи, Вестн. Сам. гос. техн. ун-та. Сер. Физ.мат. науки, 2004, выпуск 30, 170-177

DOI: https://doi.org/10.14498/vsgtu319

Использование Общероссийского математического портала Math-Net.Ru подразумевает, что вы прочитали и согласны с пользовательским соглашением

http://www. mathnet.ru/rus/agreement

Параметры загрузки:

IP : 35.174 .16 .151

26 апреля 2023 г., 13:06:42 


\section{МАТЕМАТИЧЕСКОЕ МОДЕЛИРОВАНИЕ ПРОДОЛЬНЫХ ТОКОВ СМЕЩЕНИЯ И ПОВЕРХНОСТНОГО ЭФФЕКТА В МНОГОСЛОЙНОЙ ЗЕМЛЕ И ПРОВОДАХ ЛИНИЙ ЭЛЕКТРОПЕРЕДАЧИ}

Рассматриваются основные положения математического моделирования анализа электромагнитных квазистационарных процессов в многокомпонентных системах и устройствах на примере исследования поверхностного эффекта в проводах многопроводной воздушной линии электропередачи и проводящей земле, имеющей неоднородную горизонтальную многослойную структуру при пренебрежении составляющими векторов напряженностей: продольной для магнитного и поперечной для электрического полей .

Математическое описание распространения электромагнитных волн в многокомпонентных системах и устройствах, таких, как многопроводные воздушные и кабельные линии, заземляющие устройства, линии и кабели связи и др., основано на анализе систем уравнений Максвелла, построенных для соответствующих электромагнитных полей [1]. При этом должны быть удовлетворены граничные условия на поверхностях раздела сред с различными характеристиками (воздух, земля с неоднородной структурой, провода, грозозащитные тросы, металлические конструкции и т.Д.).

Решение названных задач в подавляющем большинстве случаев производится с помощью классического перехода от дифференциального описания в рамках теории электромагнитных полей к интегральным представлениям с помощью схем замещения в виде цепей с сосредоточенными и распределенными параметрами. Эти параметры и позволяют интегрально учесть, с одной стороны, физические условия, разнообразные технические условия, среды и границы между ними. С другой стороны, они позволяют определить в названных технических системах соответствующие реакции в виде токов, напряжений, мощностей и других параметров стационарных и переходных режимов, что необходимо для принятия технических решений [2].

Обобщенное представление квазистационарных и импульсных процессов в названных выше многокомпонентных системах производится с помощью телеграфных уравнений, решение которых необходимо производить в очень широком диапазоне частот. Одним из наиболее значимых физических процессов при этом является поверхностный эффект в проводах и земле [3], имеющей неоднородную, чаще всего, слоистую структуру.

Рассмотрим учёт этого эффекта для многокомпонентных систем, не умаляя общности рассуждений, на примере многопроводной воздушной линии электропередачи. Отметим, что способы и методика решений с необходимыми изменениями могут быть использованы для других многокомпонентных систем и устройств, в частности, заземляющих конструкций и др.

Для многопроводной линии [4] названное влияние поверхностного эффекта в однородной земле и проводах учитывается в матрице продольных сопротивлений добавкой сопротивлениям идеальной линии без потерь $\Delta \boldsymbol{Z}=1 / 2 p \mu \boldsymbol{F} / \pi$ в первом приближении (с допущениями о пренебрежении составляющими векторов напряженностей: продольной для магнитного и поперечной для электрического полей) с помощью интегралов Карсона [3]. В этом случае матрица $\boldsymbol{F}$ имеет вид

$$
F=F_{z}+F_{n},
$$

где $\boldsymbol{F}_{z}$ - квадратная матрица интегралов Карсона, в которой элементы $f z_{\kappa m}$ ( $к$ и $m$ - номера проводов многопроводной линии) определяются с помощью интегральных выражений

$$
f z_{\kappa m}(r, \theta)=\int_{0}^{\infty} \frac{2 e^{-\lambda\left(h_{k}+h_{m}\right)} \cos \lambda a_{\kappa m}}{\lambda+\sqrt{\lambda^{2}+p \mu_{0} \gamma_{z}}} d \lambda ;
$$

$\boldsymbol{F}_{n}$ - диагональная матрица, собственные элементы которой определяются в виде [4]

$$
f n_{\kappa \kappa}=\frac{\mu_{\kappa} k_{c \kappa} I_{0}\left(\psi_{\kappa}\right)}{\mu_{0} \psi_{\kappa} I\left(\psi_{\kappa}\right)}
$$


В выражении (2) $r=\sqrt{\left[\left(h_{k}+h_{m}\right)^{2}+a^{2}\right] p \mu_{0} \gamma_{z}}$ и $\theta=\operatorname{arctg}\left|a_{k m} /\left(h_{k}+h_{m}\right)\right|$ - параметры интегралов Карсона, зависящие от геометрических и электромагнитных характеристик линии, таких, как $h_{k}, h_{m}, a_{k m}$ - соответственно, высоты над горизонтальной землей $k$-го и $m-$-го проводов и расстояние между ними по горизонтали, $\mu_{0}$ - магнитная проницаемость, $\rho_{z}=1 / \gamma_{z}-$ удельные сопротивление и проводимость земли, $p$ - оператор интегрального преобразования Лаплаca.

Выражение (3) учитывает поверхностный эффект [4] в $\kappa$ - ом проводе. В нём $\psi_{k}=r_{\kappa} \sqrt{p \mu_{\kappa} \gamma_{k}}$ - аргумент модифицированных функций Бесселя $I_{0}\left(\psi_{\kappa}\right), I_{1}\left(\psi_{\kappa}\right), \rho_{\kappa}=1 / \gamma_{\mathrm{\kappa}}$ удельное сопротивление и проводимость, $\mu_{\kappa}$ - магнитная проницаемость (при пренебрежении намагничиванием стальной части провода), $\kappa_{c \kappa}-$ коэффициент, приближенно отражающий многожильность, скрутку повивов провода, окисление поверхности и т.д., $r_{\kappa}$ - радиус одиночного провода (для расщепленных проводов эквивалентный радиус рассчитывается по формуле $r_{\kappa \ni}=\sqrt{r_{\kappa} a_{p}^{s-1}}$, где $r_{\kappa}$ - радиус одной из $s$ составляющих провода, расщепленного с шагом $\left.a_{p}[2]\right)$.

Вычисление элементов матрицы $F_{n}$ по формуле (3) упрощается тем, что при значениях аргумента $|\psi|>20$ с погрешностью не более $10^{-4}$ можно принять $I_{0}(\psi) / I_{l}(\psi) \approx 1$ [5].

Выражение (2) справедливо при пренебрежении токами смещения для однородной земли. Однако исследования показывают, что массивы земли часто неоднородны, причем самой распространенной является горизонтально-слоистая структура. В частности, для северных районов страны характерны малое удельное сопротивление верхнего слоя грунта и очень большое (до 10000 Ом'м и более) - для нижнего слоя вечной мерзлоты.

Для учета этого фактора при расчете продольных сопротивлений (матрицы $\boldsymbol{F}$ ) необходимо иметь интегральные выражения вида (2), аналогичные решению Карсона [3]. Получим эти выражения при допущениях Карсона для наиболее распространенного случая, когда земля состоит из двух слоев (диэлектрические, магнитные проницаемости и удельные сопротивления и проводимости соответственно $\left.\varepsilon_{I}, \varepsilon_{I I}, \mu_{I}, \mu_{I I}, \rho_{I}=1 / \gamma_{I}, \rho_{I I}=1 / \gamma_{I I}\right)$, граница между которыми лежит на глубине $d$.

Будем характеризовать волны, распространяющиеся по линии (её для упрощения изложения будем считать пока однопроводной) вдоль оси $X$, токи, заряды и поле общим динамическим гармоническим (при $p=j \omega)$ коэффициентом $e^{-\Gamma x+j \omega t}$ ( $\Gamma$ - постоянная распространения), который для удобства может быть опущен в формулах. Это предложение соответствует допущению о наличии синусоидальных волн, распространяющихся только в сторону положительной оси $X$, и отсутствии встречных волн. При этом общее решение волнового уравнения, вытекающего из системы уравнений Максвелла, составленной для данных условий, может быть записано, например, для продольной электрической напряженности в земле в следующем виде:

$\left\{\begin{array}{l}E_{x}^{I}=-\int_{0}^{\infty} M_{I}^{\prime}(\lambda) e^{\eta_{I} z} \cos \lambda y d \lambda-\int_{0}^{\infty} M_{I}^{\prime \prime}(\lambda) e^{-\eta_{I} z} \cos \lambda y d \lambda \text { для } 0 \geq Z \geq-d ; \\ E_{x}^{I I}=-\int_{0}^{\infty} M_{I I}^{\prime}(\lambda) e^{\eta_{I I} z} \cos \lambda y d \lambda \quad \text { для }-d \geq Z \geq-\infty, \\ \text { где } \eta_{I}=\sqrt{\lambda^{2}+j \omega \mu_{I} \gamma_{I}}+\omega\left(\mu_{0} \varepsilon_{0}-\mu_{I} \varepsilon_{I}\right)=\sqrt{\lambda^{2}-m_{I}^{2}} ;\end{array}\right.$
$\eta_{I I}=\sqrt{\lambda^{2}+j \omega \mu_{I I} \gamma_{I I}}+\omega\left(\mu_{0} \varepsilon_{0}-\mu_{I I} \varepsilon_{I I}\right)=\sqrt{\lambda^{2}-m_{I I}^{2}} ; M_{I}^{\prime}(\lambda), M_{I}^{\prime \prime}(\lambda), M_{I I}(\lambda)-$ произвольные функции $\lambda$, определяемые из граничных условий.

Конструкция выражений (4) обуславливается симметрией поля относительно оси $Z$, необходимостью обращения в нуль $E_{x}^{I}$ при $Z=-\infty$ и ограниченностью первого слоя земли (две составляющих в $E_{x}^{I}$ ). Кроме того, построение выражений для $\eta_{I}, \eta_{I I}$ позволяет учесть продольные токи смещения в земле.

Полагая по Карсону [3], что в земле поперечные составляющие $E_{z}$ и $E_{y}$ незначительны по сравнению с $E_{x}$, можно получить из уравнения Максвелла rot $\boldsymbol{E}=-\mu \frac{\partial}{\partial t} \boldsymbol{H}$ с учетом (4) следующие соотношения: 


$$
\left\{\begin{array}{l}
H_{y}^{I}=\frac{1}{j \omega \mu_{I}}\left[\int_{0}^{\infty} \eta_{I} M_{I}^{\prime}(\lambda) e^{\eta_{I} z} \cos \lambda y d y-\int_{0}^{\infty} \eta_{I} M_{I}^{\prime \prime}(\lambda) e^{-\eta_{I} z} \cos \lambda y d \lambda\right] \\
H_{z}^{I}=\frac{1}{j \omega \mu_{I}}\left[\int_{0}^{\infty} \lambda M_{I}^{\prime}(\lambda) e^{\eta_{I} z} \cos \lambda y d y-\int_{0}^{\infty} \lambda M_{I}^{\prime \prime}(\lambda) e^{-\eta_{I} z} \cos \lambda y d \lambda\right], \\
H_{y}^{I I}=\frac{1}{j \omega \mu_{I I}} \int_{0}^{\infty} \eta_{I I} M_{I I}(\lambda) e^{\eta_{I I} z} \cos \lambda y d \lambda, \\
H_{z}^{I I}=\frac{1}{j \omega \mu_{I I}} \int_{0}^{\infty} \lambda M_{I I}(\lambda) e^{\eta_{I I} z} \cos \lambda y d \lambda .
\end{array}\right.
$$

Представим поле в воздухе состоящим из двух составляющих, например

$$
H_{y}=H_{y}^{0}+H_{y}^{\prime}, H_{z}=H_{z}^{0}+H_{z}^{\prime},
$$

где $H_{y}^{0}, H_{z}^{0}$ определяют поле от тока $I$ в проводе, а $H_{y}^{\prime}, H_{z}^{\prime}$ - поле от тока в земле.

Теперь, считая распределение тока по сечению провода симметричным, можно записать на основании закона полного тока для любой точки в воздухе с координатами $y, z$ :

$$
H_{y}^{0}=\frac{\cos \theta}{d} \frac{I}{2 \pi}, H_{r}^{0}=\frac{\sin \theta}{d} \frac{I}{2 \pi},
$$

где $d=\sqrt{y^{2}+(h-z)^{2}}, \cos \theta=\frac{h-z}{d}, \sin \theta=\frac{y}{d}$.

В частности, на границе раздела $Z=0, H_{y}^{0}$ и $H_{z}^{0}$ могут быть выражены с помощью интегралов Фурье

$$
H_{\left.y\right|_{z=0}}^{0}=\frac{I}{2 \pi} \int_{0}^{\infty} \cos \lambda y e^{-\lambda h} d \lambda, H_{\left.z\right|_{z=0}}^{0}=\frac{I}{2 \pi} \int_{0}^{\infty} \sin \lambda y e^{-\lambda h} d \lambda .
$$

Вторичное магнитное поле в воздухе, так же как и в [3], может быть принято в виде

$$
H_{y}^{\prime}=\int_{0}^{\infty} \Phi(\lambda) \cos \lambda y e^{-\lambda z} d \lambda, H_{z}^{\prime}=\int_{0}^{\infty} \Phi(\lambda) \sin \lambda y e^{-\lambda z} d \lambda .
$$

На поверхностях раздела сред с различными характеристиками нормальные и тангенциальные составляющие $H_{y}$ и $H_{z}$ должны удовлетворять следующим условиям:

$$
\begin{gathered}
H_{y_{b}}=H_{y}^{I}, \quad \mu_{0} H_{z b}=\mu_{I} H_{z} \text { для } z=0 ; \\
H_{y}^{I}=H_{y}^{I I}, \mu_{I} H_{z}=\mu_{I I} H_{z} \text { для } z=-d .
\end{gathered}
$$

Это позволяет составить систему для определения неизвестных функций $M_{I}^{\prime}(\lambda), M_{I}^{\prime \prime}(\lambda)$, $M_{I I}(\lambda), \Phi(\lambda)$ на основании выражений (5-9):

$$
\left\{\begin{array}{c}
\frac{1}{j \omega \mu_{I}} \eta_{I} M_{I}^{\prime}(\lambda)-\frac{1}{j \omega \mu_{I}} \eta_{I} M_{I}^{\prime \prime}(\lambda)=\frac{I}{2 \pi} e^{-\lambda h}+\Phi(\lambda) \\
\mu_{0}\left[\frac{1}{j \omega \mu_{I}} \lambda M_{I}^{\prime}(\lambda)+\frac{1}{j \omega \mu_{I}} \lambda M_{I}^{\prime \prime}(\lambda)\right]=\mu_{I}\left[\frac{I}{2 \pi} e^{-\lambda h}-\Phi(\lambda)\right] \\
\eta_{I} M_{I}^{\prime}(\lambda) e^{-\eta_{I} d}-\eta_{I} M_{I}^{\prime \prime}(\lambda) e^{\eta_{I} d}=\eta_{I I} M_{I I}(\lambda) e^{-\eta_{I I} d} \\
\mu_{I}\left[M_{I}^{\prime}(\lambda) e^{-\eta_{I} d}+M_{I}^{\prime \prime}(\lambda) e^{\eta_{I} d}\right]=\mu_{I I} M_{I I}(\lambda) e^{-\eta_{I I} d}
\end{array}\right.
$$

Решая систему (11) для часто встречающегося случая $\mu_{I}=\mu_{I I}=\mu_{0}$, получим выражения для функций $M_{I}^{\prime}(\lambda), M_{I}^{\prime \prime}(\lambda), M_{I I}(\lambda), \Phi(\lambda)$ в виде 


$$
\begin{gathered}
M_{I}^{\prime}(\lambda)=\frac{j \omega \mu_{0}}{2 \pi \chi_{1}} I e^{\eta_{I} d-\lambda h}\left(\eta_{I}+\eta_{I I}\right), M_{I}^{\prime \prime}(\lambda)=\frac{j \omega \mu_{0}}{2 \pi \chi_{1}} I e^{-\eta_{I} d-\lambda h}\left(\eta_{I}-\eta_{I I}\right), \\
M_{I I}(\lambda)=\frac{j \omega \mu_{0}}{\pi x_{1}} I \eta_{I} e^{\eta_{I I} d-\lambda h}, \Phi(\lambda)=\frac{\chi_{z}}{2 \pi \chi_{1}} I e^{-\lambda h},
\end{gathered}
$$

где $\chi_{1}=\left(\eta_{I}^{2}+\eta_{I I} \lambda\right) \operatorname{sh} \eta_{I} d+\left(\eta_{I} \eta_{I I}+\eta_{I} \lambda\right) \operatorname{ch} \eta_{I} d, \chi_{2}=\left(\eta_{I}^{2}-\eta_{I I} \lambda\right) \operatorname{sh} \eta_{I} d+\left(\eta_{I} \eta_{I I}-\eta_{I} \lambda\right) \operatorname{ch} \eta_{I} d$. $\mathrm{C}$ помощью этих выражений могут быть записаны все интересующие нас составляющие поля в каждом слое земли.

Для дальнейшего анализа нам понадобятся продольная составляющая $E_{x}^{I}$ вектора электрической напряженности и тангенциальная составляющая $H_{y \varepsilon}$ вектора магнитной напряженности:

$$
\begin{gathered}
E_{x}^{I}=-\frac{j \omega \mu}{\pi} I \int_{0}^{\infty} \frac{e^{-\lambda h} \cos \lambda y}{x_{1}}\left[\eta_{I} \operatorname{ch} \eta_{I}(z+d)+\eta_{I I} \operatorname{sh} \eta_{I}(z+d)\right] d \lambda, \\
H_{y b}=H_{y}^{0}+H_{y}^{\prime}=\frac{\cos \theta}{d} \frac{I}{2 \pi} \int_{0}^{\infty} \frac{x_{2}}{x_{1}} e^{-(h+z)} \cos \lambda y d \lambda .
\end{gathered}
$$

Для определения продольной составляющей $E_{x \beta}$ электрической напряженности в воздухе используем известное выражение

$$
E_{x \theta}=-j \omega A_{x \theta}-\frac{\partial}{\partial x} V,
$$

где $A_{x в}$ - продольная составляющая векторного потенциала, $V$ - скалярный потенциал.

Составляя теперь разность величин, входящих в $(15)$, для точек $(y, z)$ и $(y, 0)$ на поверхности раздела $Z=0$, а также учитывая, что

$$
A_{x b}(y, z)-A_{x b}(y, 0)=\mu_{0} \int_{0}^{z} H_{y b}(y, z) d z
$$

можем записать

$$
E_{x \theta}=E_{x \varepsilon}(y, 0)-\mu_{0} \int_{0}^{z} H_{y b}(y, z) d z-\frac{\partial}{\partial z} U
$$

где $U$ - разность потенциалов $V(y, z)$ и $V(y, 0)$.

Интеграл (17), вычисленный с учетом (14), состоит из двух слагаемых:

$$
\mu_{0} \int_{0}^{z} H_{y b}(y, z) d z=\frac{\mu_{0}}{2 \pi} I \ln \frac{\sqrt{y^{2}+h^{2}}}{\sqrt{y^{2}+(h-z)^{2}}}+\frac{\mu_{0}}{2 \pi} \int_{0}^{\infty} \frac{e^{-\lambda h} \cos \lambda y \chi_{2}}{\chi_{1} \lambda}\left(1-e^{-\lambda z}\right) .
$$

Подставляя теперь (13) и (18) в ( 17), получим

$$
\begin{gathered}
E_{x b}=-\frac{j \omega \mu_{0}}{2 \pi} I \int_{0}^{\infty} \frac{e^{-\lambda h}-e^{-\lambda(h+z)}}{\lambda} \cos \lambda y d \lambda-\frac{j \omega \mu_{0}}{\pi} I \int_{0}^{\infty} \frac{\eta_{I I} \operatorname{sh} \eta_{I} d+\eta_{I} \operatorname{ch} \eta_{I} d}{\chi_{1}} e^{-(h+z)} \cos \lambda y d \lambda- \\
-\frac{j \omega \mu_{0}}{2 \pi} I \ln \frac{\sqrt{y^{2}+h^{2}}}{\sqrt{y^{2}+(h-z)^{2}}}-\frac{\partial}{\partial z} U
\end{gathered}
$$

Здесь первый интеграл в правой части представляет собой разновидность интеграла Фруллани [5] $\int_{0}^{\infty} \frac{e^{-\lambda h}-e^{-\lambda(h+z)}}{\lambda} \cos \lambda y d y=\ln \sqrt{\frac{y^{2}+(h+z)^{2}}{\sqrt{y^{2}+h^{2}}}}$. Окончательно, после упрощений, (19) можно записать в виде

$$
E_{x b}=-\frac{j \omega \mu}{\pi} I\left[\int_{0}^{\infty} \frac{e^{-\lambda(h+z)} \cos \lambda y}{\lambda+A(\lambda)} d \lambda+\ln \frac{D}{d}\right]-\frac{\partial}{\partial z} V
$$


где $A(\lambda)=\frac{\eta_{I}\left(\eta_{I \mathrm{II}}+\operatorname{cth} \eta_{I} d\right)}{1+\eta_{I \mathrm{II}} \mathrm{cth} \eta_{I} d}, \eta_{I \mathrm{II}}=\frac{\eta_{I}}{\eta_{I I}}, D=\sqrt{y^{2}+(h+z)^{2}}, d=\sqrt{y^{2}+h^{2}}$.

Откуда, приравнивая продольную напряженность на поверхности провода найденному выражению (20), получим аналогично (2) выражение для учета влияния двухслойной земли и продольных токов смещения в матрице сопротивлений линии, выведенное с допущениями Карсона:

$$
f_{z_{k m}}=\int_{0}^{\infty} \frac{2 e^{-\lambda\left(h_{k}+h_{m}\right)} \cos \lambda a_{\kappa m}}{\lambda+A(\lambda)} d \lambda .
$$

Более точное решение этой задачи можно построить при использовании методики Гринберга и Бонштедта [6].

Рассмотренный метод учета влияния двухслойной земли в матрице продольных сопротивлений обладает большой наглядностью. Однако для многослойной земли выкладки становятся весьма сложными. Поэтому целесообразно рассмотреть метод, позволяющий решать такую задачу. В этом случае выражения, аналогичные $E_{x}^{I}(4)$, должны быть записаны для $n-1$ верхних слоев и аналогичные $E_{x}^{I I}$ для последнего. Это позволит так же, как и в (5), определить общий вид $H_{x}$ и $H_{y}$ для всех слоев, и построить систему граничных условий типа (10) для всех границ раздела $Z_{i}=0,1,2, \ldots, n-1=-d_{i}\left(Z_{0}=0\right)$. Если, как и ранее, положить $\mu_{i}=\mu_{0}(i=1,2, \ldots, n)$, то система уравнений для определения функций $M_{i}^{\prime}(\lambda), M_{i}^{\prime \prime}(\lambda), \Phi(\lambda)$ примет вид:

$$
\left\{\begin{array}{l}
\frac{\eta_{I}}{j \omega \mu_{0}} M_{1}^{\prime}(\lambda)-\frac{\eta_{I}}{j \omega \mu_{0}} M_{1}^{\prime \prime}(\lambda)=\frac{I}{2 \pi} e^{-\lambda h}+\Phi(\lambda) ; \\
\frac{\lambda}{j \omega \mu_{0}} M_{1}^{\prime}(\lambda)+\frac{\lambda}{j \omega \mu_{0}} M_{1}^{\prime \prime}(\lambda)=\frac{I}{2 \pi} e^{-\lambda h}-\Phi(\lambda) ; \\
\eta_{I} M_{1}^{\prime}(\lambda) e^{-\eta_{I} d_{I}}-\eta_{I} M_{1}^{\prime \prime}(\lambda) e^{\eta_{I} d_{I}}=\eta_{I I} M_{2}^{\prime}(\lambda) e^{-\eta_{I I} d_{I}}-\eta_{I I} M_{2}^{\prime \prime}(\lambda) e^{\eta_{I I} d_{I}} ; \\
M_{1}^{\prime}(\lambda) e^{-\eta_{I} d_{I}}+M_{1}^{\prime \prime} e^{\eta_{I} d_{i}}=M_{2}^{\prime}(\lambda) e^{-\eta_{I I} d_{I}}+M_{2}^{\prime \prime}(\lambda) e^{\eta_{I I} d_{I}} ; \\
\ldots \ldots \ldots \ldots \ldots \ldots \ldots \ldots \ldots \ldots \ldots \ldots \ldots \ldots \ldots \ldots \ldots \ldots \ldots \ldots \ldots \ldots \ldots \ldots \ldots \ldots \ldots \ldots \ldots \ldots \ldots \ldots \\
\eta_{i} M_{i}^{\prime}(\lambda) e^{-\eta_{i} d_{i}}-\eta_{i} M_{i}^{\prime \prime}(\lambda) e^{\eta_{i} d_{i}}=\eta_{i+1} M_{i+1}^{\prime}(\lambda) e^{-\eta_{i+1} d_{i}}-\eta_{i+1} M_{i+1}^{\prime \prime}(\lambda) e^{\eta_{i+1} d_{i}} \\
M_{i}^{\prime}(\lambda) e^{-\eta_{i} d_{i}}+M_{i}^{\prime \prime} e^{\eta_{i} d_{i}}=M_{i+1}^{\prime}(\lambda) e^{-\eta_{i+1} d_{i}}+M_{i+1}^{\prime \prime}(\lambda) e^{\eta_{i+1} d_{i}} ; \\
\eta_{n-1} M_{n-1}^{\prime}(\lambda) e^{-\eta_{n-1} d_{n-1}}-\eta_{n-1} M_{n-1}^{\prime \prime}(\lambda) e^{-\eta_{n-1} d_{n-1}}=\eta_{n} M_{n}^{\prime}(\lambda) e^{-\eta_{n} d_{n-1}} ; \\
M_{n-1}^{\prime}(\lambda) e^{-\eta_{n-1} d_{n-1}}+M_{n-1}^{\prime \prime} e^{-\eta_{n-1} d n-1}=M_{n}^{\prime}(\lambda) e^{-\eta_{n} d_{n-1}} .
\end{array}\right.
$$

Для каждой границы раздела составлено по два уравнения. Меняя знаки в каждом первом из двух (кроме уравнения границы $Z=0$ ) и деля на его левую и правую части соответствующие части второго уравнения, получим после преобразований:

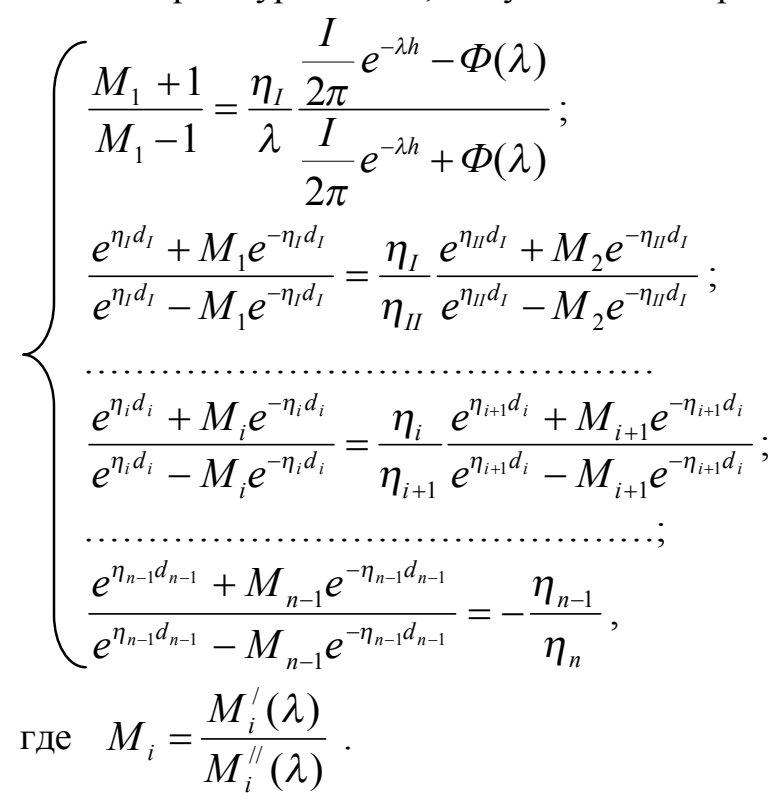


Система (23) (за исключением первого уравнения) может быть представлена в виде:

$$
\left\{\begin{array}{l}
\operatorname{cth}\left(\eta_{i} d_{i}-\frac{1}{2} \ell n M_{i}\right)=\frac{\eta_{i}}{\eta_{i+1}} \operatorname{cth}\left(\eta_{i+1} d_{i}-\frac{1}{2} \ln M_{i+1}\right), i=1,2, \ldots \ldots n-2 ; \\
\operatorname{cth}\left(\eta_{n-1} d_{n-1}-\frac{1}{2} \ln M_{n-1}\right)=-\frac{\eta_{n-1}}{\eta_{n}}
\end{array}\right.
$$

откуда можно выразить

$$
\frac{1}{2} \ln M_{n-1}=\eta_{n-1} d_{n-1}+\operatorname{arcth} \frac{\eta_{n-1}}{\eta_{n}}
$$

и подставить полученное выражение в предыдущие уравнения. Проделав эти операции для всех $M_{i}$, получим окончательно из первого уравнения (24) соотношение

$$
\frac{1}{2} \ln M_{1}=\eta_{I} \Delta d_{I}+\operatorname{arcth}\left\{\frac{\eta_{I}}{\eta_{I I}} \operatorname{cth}\left\{\eta_{I I} \Delta d_{I I}+\ldots+\operatorname{arcth} \frac{\eta_{n-1}}{\eta_{n}}\right\} \ldots\right\}=\chi,
$$

где $\Delta d_{i}=d_{i}-d_{i-1}$, что позволяет определить

$$
M_{1}=\frac{M_{1}^{\prime}(\lambda)}{M_{1}^{\prime \prime}(\lambda)}=e^{2 \chi}
$$

Используя теперь (27) в первом уравнении системы (23), получим

$$
\operatorname{cth} \chi=\frac{\frac{I}{2 \pi} e^{-\lambda h}-\Phi(\lambda)}{\frac{I}{2 \pi} e^{-\lambda h}+\Phi(\lambda)} \frac{\eta_{I}}{\lambda},
$$

откуда

$$
\Phi(\lambda)=\frac{I}{2 \pi} e^{-\lambda h} \frac{\eta_{I}-\lambda \operatorname{cth} \chi}{\eta_{I}+\lambda \operatorname{cth} \chi} .
$$

Подставляя $\Phi(\lambda)$ в два первых уравнения (22), определим $M_{1}^{\prime}(\lambda)$ и $M_{1}^{\prime \prime}(\lambda)$ :

$$
M_{1}^{\prime}(\lambda)=\frac{j \omega \mu_{0}}{2 \pi} e^{-\lambda h} \frac{\operatorname{cth} \chi+1}{\lambda \operatorname{cth} \chi+\eta_{I}}, M_{1}^{\prime \prime}(\lambda)=\frac{j \omega \mu_{0}}{2 \pi} e^{-\lambda h} \frac{\operatorname{cth} \chi+1}{\lambda \operatorname{cth} \chi+\eta_{I}} .
$$

Теперь можно последовательно найти все остальные неизвестные в системе (22), записать выражения для $E_{x}^{I}$ и $H_{y b}$ с помощью выражений, аналогичных (16-19), и определить $E_{x в}$ в виде

$$
E_{x \theta}=-\frac{j \omega \mu_{0}}{\pi} I \int_{0}^{\infty} \frac{\operatorname{cth} \chi}{\eta_{I}+\lambda \operatorname{cth} \chi} e^{-\lambda(h+z)} \cos \lambda y d \lambda-\frac{j \omega \mu_{0}}{2 \pi} I \ln \frac{D}{d}-\frac{\delta}{\delta z} V .
$$

Это позволяет записать выражение, учитывающее в матрице продольных сопротивлений влияние многослойной земли, вид которого совпадает с (21) при $A(\lambda)=\eta_{I}$ th $\chi$, где $\chi$ определяется из (26):

$$
f_{z_{\kappa m}}=\int_{0}^{\infty} \frac{2 e^{-\lambda\left(h_{\kappa}+h_{m}\right)} \cos \lambda a_{\kappa m}}{\lambda+\eta_{I} \operatorname{th} \chi} d \lambda
$$

В частности, для случая, когда земля может быть представлена двумя слоями и в диапазоне частот $10^{5}-10^{7}$ Гц проникновением волны в глубокие слои можно пренебречь (первый слой с конечной толщиной, а второй - не ограничен) можно после преобразований записать для $A(\lambda)$ выражение:

$$
A(\lambda)=\eta_{I} \operatorname{cth}\left(\lambda_{I} \Delta d_{I}+\operatorname{arcth} \frac{\eta_{I}}{\eta_{I I}}\right)=\frac{\eta_{I}\left(\eta_{I \mathrm{II}}+\operatorname{cth} \eta_{I} d_{I}\right)}{1+\eta_{I \mathrm{II}} \operatorname{cth} \eta_{I} d_{I}} .
$$

Соотношение (33) тождественно тому, что получили для $A(\lambda)$ Ведепол и Весли [7], но более удобно для численных расчетов. 

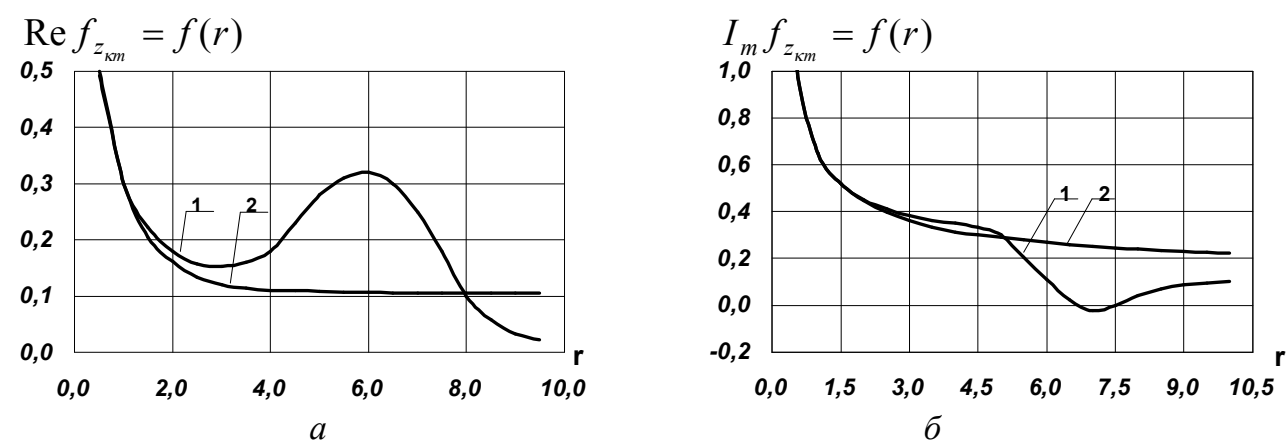

Р и с. 1. Зависимости:

$a-\operatorname{Re} f_{z_{\kappa m}}=f(r) ; \sigma-I_{m} f_{z_{\kappa n}}=f(r) ; 1-$ с учетом токов смещения; 2 - без учета токов смещения; $\eta_{\mathrm{I}} / \eta_{\mathrm{II}}=10 ; \theta=0^{\circ} ; d_{I}=15 \mathrm{M}$

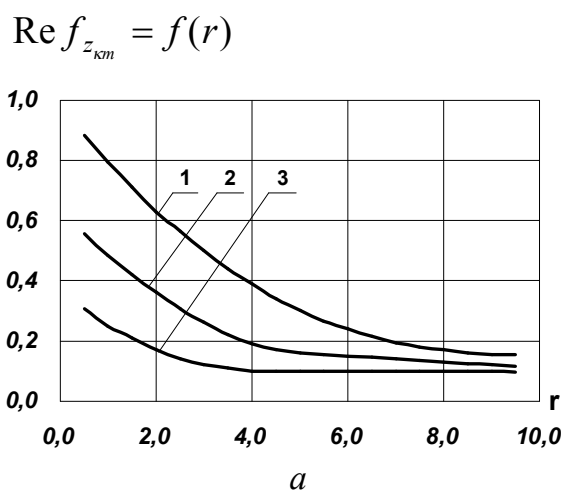

$I_{m} f_{z_{\kappa m}}=f(r)$

Р и с. 2. Зависимости:

$a-\operatorname{Re} f_{z_{k m}}=f(r) ; \sigma-I_{m} f_{z_{k m}}=f(r) ; 1-$ при $\eta_{\mathrm{I}} / \eta_{\text {II }}=0,1 ; 2-$ при $\eta_{\mathrm{I}} / \eta_{\mathrm{II}}=1 ;$

$3-$ при $\eta_{\mathrm{I}} / \eta_{\text {II }}=10 ; \quad \theta=0^{\circ} ; \quad d_{I}=15$ м. Токи смещения в расчете не учитывались

$\operatorname{Re} f_{z_{x n}}=f(r)$

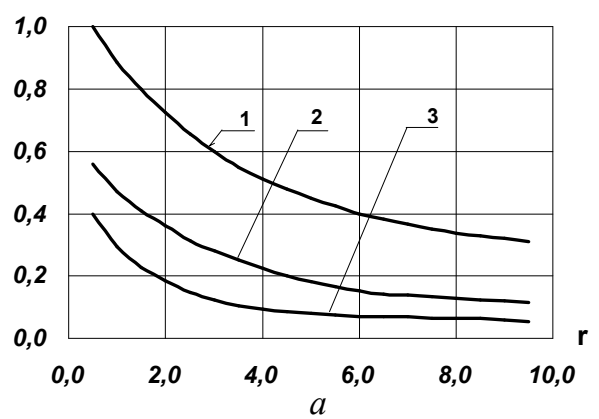

$$
I_{m} f_{z_{k n}}=f(r)
$$

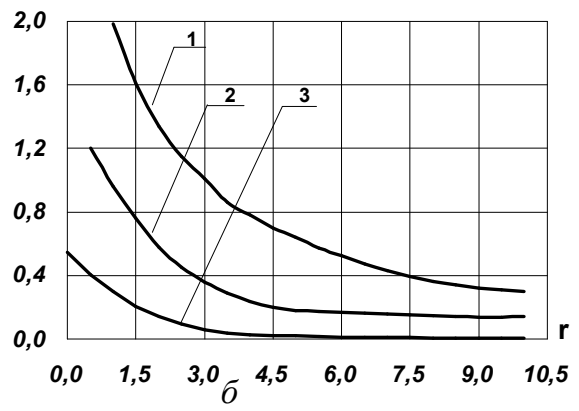

Р и с. 3. Зависимости:

$a-\operatorname{Re} f_{z_{к m}}=f(r) ; \sigma-I_{m} f_{z_{к n}}=f(r) ; 1$ - при $\rho_{I} / \rho_{I I}=0,1 ; 2$ - при $\rho_{I} / \rho_{I I}=1 ;$

3 - при $\rho_{I} / \rho_{I I}=10 ; \quad \theta=60^{\circ} ; \quad d_{I}=15$ м. Токи смещения в расчете не учитывались

$\operatorname{Re} f_{z_{x n}}=f(r)$

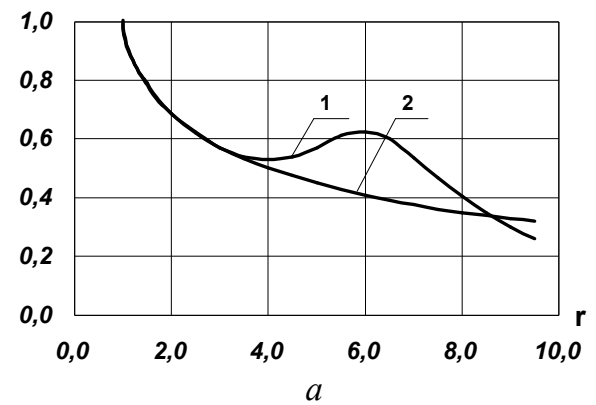

$I_{m} f_{z_{k n}}=f(r)$

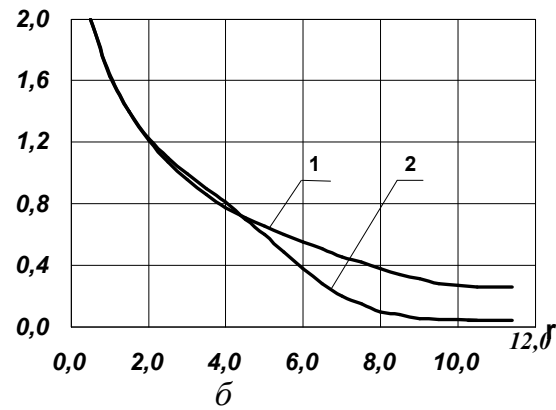

Р и с. 4. Зависимости:

$a-\operatorname{Re} f_{z_{\kappa m}}=f(r) ; \sigma-I_{m} f_{z_{\kappa n}}=f(r) ; 1-$ с учетом токов смещения; 2- без учета токов смещения; при $\rho_{I} / \rho_{I I}=0,1 ; \theta=60^{\circ} ; d_{I}=15 \mathrm{M}$ 
Нетрудно установить его связь с выражениями, полученными Карсоном. В самом деле, для однородной земли $A(\lambda)=\eta_{I}=\sqrt{\lambda^{2}+p \mu_{I} \gamma_{I}-p^{2}\left(\mu_{0} \varepsilon_{0}-\mu_{I} \varepsilon_{I}\right)}$, что эквивалентно результатам Уайза [8], уточняющим интеграл Карсона при $\mu_{I} \varepsilon_{I} \neq \mu_{0} \varepsilon_{0}$ (учет продольных токов смещения), а при $\mu_{I} \varepsilon_{I}=\mu_{0} \varepsilon_{0}$ получаем совпадение с выражением (2).

На рис. 1-4 представлены результаты расчетов интегралов (33) с учётом (34) при некоторых конкретных соотношениях электрических и магнитных характеристик слоев двухслойной земли. Анализ численных результатов расчетов показывает, что влияние двухслойности земли в основном сказывается при малых частотах (им соответствуют малые значения параметра $r$ ). Отметим, что для двухслойной земли параметры $r$ и $\theta$ вычисляются аналогично случаю однородной земли с характеристиками первого слоя. Это не имеет принципиального значения, однако удобно для сравнения результатов расчётов.

Влияние токов смещения проявляется на более высоких частотах, где вследствие их емкостного характера появляется пик в действительной части интеграла (4) и резкий спад в мнимой части. Это наиболее четко выражено для случая, когда $\rho_{I}=1 / \gamma_{I}>\rho_{I I}=1 / \gamma_{I I}$. В противоположных случаях $\left(\rho_{I}<\rho_{I I}\right)$ этот эффект проявляется незначительно при $\theta=0$ и становится существенным с ростом $\theta$.

\section{БИБЛИОГРАФИЧЕСКИЙ СПИСОК}

1. Л.Р.Нейман, К.С.Демирчян. Теоретические основы электротехники. М.: Энергоатомиздат, 2001. 320 с.

2. В.И.Идельчик. Электрические системы и сети. М.: Энергоатомиздат, 1989. 458 с.

3. J.R.Carson. Wave propagation in overhead wires with ground return// BSTJ. V. 5. №4. 1926. P. 31-37.

4. М.В.Костенко, Л.С.Перельман. К расчёту волновых процессов в многопроводных линиях//Известия АН СССР. Сер. Энергетика и транспорт. 1963. №6. С. 71-80.

5. И.С. Градитейн, И.М Рыжик. Таблицы сумм, интегралов, сумм, рядов и произведений. М.: ГИФМЛ, 1962. $388 \mathrm{c.}$

6. В.Г.Гольдштейн, Н.В.Сайдова, А.К.Танаев. Уточнённая математическая модель поверхностного эффекта в многослойной земле // Вестник СамГТУ. Серия: Физико-математические науки Вып. 19. 2003. С. 129-134.

7. L.M.Wedepohl, R.G.Wasley. Wave propagation in multiconductor lines. Calculation of series impedance for multilayer earth. Proc. IEE. V. 113. №4. 1966. P. 36-42.

8. W.H.Wise. Propagation of high frequency currents// Proc. IRE. V.22. 1934. P. 29-37. 